\title{
An Analytical Representation of the 3GPP 3D Channel Model Parameters for mmWave Bands
}

\author{
Margarita Gapeyenko, Vitaly Petrov, \\ Dmitri Moltchanov, Sergey Andreev, \\ Yevgeni Koucheryavy, Mikko Valkama \\ Tampere University of Technology, Finland
}

\begin{abstract}
Accurate performance prediction for the emerging 3GPP New Radio (NR) technology over millimeter-wave (mmWave) bands is crucial for the upcoming deployments of $5 \mathrm{G}$ and beyond cellular networks, utilizing NR. 3GPP has recently presented a 3D multipath cluster-based mmWave channel model for $5 \mathrm{G}$ NR, which captures the salient propagation characteristics of the mmWave bands, allowing for better prediction of mmWave system performance. However, it is difficult to directly employ the 3GPP models for analytical system characterization, as most of the parameters are computed through iterative algorithms. In the paper, we address this problem by presenting a statistical approximation for the important parameters of the 3GPP 3D cluster-based channel model, particularly, zenith angle of arrival and power share of every cluster. We then show how the constructed approximation can be used to analytically derive the performance indicators for mmWave NR systems, including outage probability. We compare the results obtained with our proposed statistical approximation model with those given by stateof-the-art simplified single cluster analytical models as well as illustrate the improvements in the accuracy of results.
\end{abstract}

\section{KEYWORDS}

3GPP New Radio, 5G wireless communications, mmWave multipath propagation, 3D channel model

\footnotetext{
This work was supported by Intel Corporation, the Academy of Finland (projects WiFiUS and PRISMA), and by the project TAKE-5: The 5th Evolution Take of Wireless Communication Networks, funded by Tekes. The work of M. Gapeyenko has been supported by Nokia Foundation. V. Petrov acknowledges the support of HPY Research Foundation funded by Elisa.

Permission to make digital or hard copies of all or part of this work for personal or classroom use is granted without fee provided that copies are not made or distributed for profit or commercial advantage and that copies bear this notice and the full citation on the first page. Copyrights for components of this work owned by others than ACM must be honored. Abstracting with credit is permitted. To copy otherwise, or republish, to post on servers or to redistribute to lists, requires prior specific permission and/or a fee. Request permissions from permissions@acm.org.

mmNets '18, October 29, 2018, New Delhi, India

(c) 2018 Association for Computing Machinery. ACM ISBN 978-1-4503-5928-3/18/10 ..\$15.00

https://doi.org/10.1145/3264492.3264498
}

\author{
Mustafa Riza Akdeniz, Nageen Himayat \\ Intel Corporation, Santa Clara, CA, USA
}

\begin{abstract}
ACM Reference Format:
Margarita Gapeyenko, Vitaly Petrov, Dmitri Moltchanov, Sergey Andreev, Yevgeni Koucheryavy, Mikko Valkama and Mustafa Riza Akdeniz, Nageen Himayat. 2018. An Analytical Representation of the 3GPP 3D Channel Model Parameters for mmWave Bands. In 2nd ACM Workshop on Millimeter Wave Networks and Sensing Systems (mmNets '18), October 29, 2018, New Delhi, India. ACM, New York, NY, USA, 6 pages. https://doi.org/10.1145/3264492.3264498
\end{abstract}

\section{INTRODUCTION AND MOTIVATION}

While the first release of 3GPP New Radio (NR) technology specification has been completed [9], ongoing research continues to explore challenges related to advanced networking options for $5 G[7,11]$. These efforts require detailed performance evaluation and optimization models that capture the specifics of inherently complex millimeter-wave (mmWave) band propagation.

The propagation dynamics of mmWave wireless channels in realistic deployments were conventionally assessed with real measurements and/or ray-tracing simulations [2, 8]. These results were later mapped onto models for further performance assessment in realistic deployments. There are two large classes of models proposed for mmWave channels. The first one is presented in [1] and introduces the notion of clusters that describe the rays between the transmitter (Tx) and the receiver $(\mathrm{Rx})$, which experience similar propagation impairments in terms of delay, power attenuation, and geometry of arrival and departure. This model explicitly captures multipath propagation and randomness associated with each of the arriving components. The second class of the models employs "averaging" considerations for simplicity, so-called single cluster, where the impairments of the propagation environment are averaged out to provide the mean propagation losses at a certain separation distance. Both model classes also feature extensions to human body blockage effects [4].

Performance assessment of 5G NR in various deployments and use-cases is currently conducted with both analytical tools and system-level simulations (SLS). However, so far, the assumptions regarding the channel model used in such performance assessment fundamentally differ. In SLS-based studies [12], the authors chiefly rely on 3GPP 3D clusterbased channel model specified in [1] as it provides a much 
more detailed description of the propagation phenomena that explicitly captures power, delay, and geometry of each cluster arriving at the Rx. However, it comes at the expense of more complex model structure, where the resulting parameters are calculated algorithmically for a given set of environmental and system conditions. As a result, the model is not analytically tractable; therefore, the authors working with analytical modeling of $5 \mathrm{G}$ NR $[3,10]$ mainly utilize simpler models that provide "average" propagation losses (single cluster case) in typical environments, such as 3GPP UMi-street canyon path loss model for line-of-sight (LoS) and non-LoS (nLoS) from [1]. The use of "averaged" models affects the accuracy of the results and complicates the process of calibration between the SLS and analytical tools.

In this paper, aiming to improve the accuracy of analytical performance evaluation frameworks for 3GPP NR, we follow a statistical approach to develop analytical approximations for the output parameters of the 3GPP 3D cluster-based channel model in case of LoS. As an illustrative example, we focus on zenith angle of arrival as well as the associated cluster power shares, which become the parameters for our firstorder performance analysis of $5 \mathrm{G}$ mmWave cellular in urban scenarios with human body blockage. We show that these parameters follow well-known distributions for a wide range of environmental and system conditions. We also apply the proposed approximations to analytically derive the outage probability in $5 \mathrm{G}$ mmWave systems. Finally, we compare the results obtained with the proposed approximations against those given by an algorithmic implementation of the 3GPP 3D cluster-based channel model as well as those derived by employing the UMi-street canyon model (single LoS cluster) with blockage enhancements [5]. We demonstrate that our approximation model offers more accurate results as compared to the "averaged" UMi-street canyon model.

The rest of this paper is organized as follows. First, in Section 2, we describe the 3GPP 3D cluster-based channel model parameters. In Section 3, we construct our proposed analytical approximations. Finally, in Section 4, we assess the accuracy of the developed approximation as well as consider an illustrative example of the use of the formulated model. Conclusions are drawn in the last section.

\section{3GPP 3D CLUSTER-BASED MODEL}

The standardized 3GPP 3D cluster-based channel model presented in [1] assumes that the received power at the user equipment (UE) comprises power coming from a number of clusters, including LoS path and several reflected components. It thus binds the specifics of mmWave propagation environment to (i) zenith angle of arrival/departure (ZOA/ZOD) and azimuth angle of arrival/departure (AOA/AOD) of a cluster, (ii) delay of every cluster, and (iii) fraction of power contributed by a cluster. However, this model involves complex functions of random variables, which complicates its usage in applied mathematical analysis.

According to 3GPP, 3D cluster-based channel modeling can be decomposed into three steps: (i) specifying the scenario and calculating its geometry, such as LoS ZOA/ZOD and LoS AOA/AOD, (ii) deriving correlated large-scale parameters, including delay spread (DS), angular spreads, Ricean $\mathrm{K}$-factor $(K)$, and shadow fading (SF), as well as (iii) generating small-scale parameters: delay, power, $\mathrm{AOA} / \mathrm{AOD}$, and $\mathrm{ZOA} / \mathrm{ZOD}$. The procedure describing the generation of largescale parameters is given in sub-clause 3.3.1 of WINNER II Deliverable [6]. Note that the total number of clusters could be chosen within the range of 4 to 20 [1], and this will affect the values of some of the parameters of the model.

The $n$-th cluster's delay is obtained as in Section 7.5 of [1],

$$
\tau_{n}^{\prime}=-r_{\tau} \mathrm{DS} \ln \left(X_{n}\right)
$$

where $\tau^{\prime}$ is the delay before sorting procedure, $r_{\tau}$ is the delay distribution proportionality factor taken from Table 7.5-6 Part 1 of TR 38.901 [1], DS is the delay spread with mean and variance given in Table 7.5-6 Part 1 [1], while the generation procedure is described in [1], $X_{n}$ is drawn from the uniform distribution in $(0,1)$, and $n$ is the cluster index $\{1,2 \ldots N\}$. Once all the delays are calculated, they are normalized by subtracting the minimum delay and then sorted in the increasing order, $\tau_{n}=\operatorname{sort}\left[\tau_{n}^{\prime}-\min \left(\tau_{n}^{\prime}\right)\right]$.

The delays in case of LoS take the following form

$$
\tau_{n}^{\mathrm{LoS}}=\tau_{n} / C_{\tau},
$$

where $C_{\tau}=0.7705-0.0433 K+0.0002 K^{2}+0.000017 K^{3}$ and $K$ is the Ricean $\mathrm{K}$-factor expressed in $\mathrm{dB}$.

Once delays are calculated and sorted, one proceeds by specifying the received power of each cluster. The power of cluster $n, P_{n}$, is obtained at step 6 of Section 7.5 in [1] as

$$
P_{n}^{\prime}=\exp \left(-\tau_{n} \frac{r_{\tau_{n}}-1}{r_{\tau_{n}} \mathrm{DS}}\right) 10^{\frac{-Z_{n}}{10}},
$$

where $Z_{n}$ captures the effect of per-cluster shadowing and follows a normal distribution with the zero mean and variance $\sigma^{2}$. The cluster powers in LoS conditions are given as

$$
P_{n}=\frac{1}{K_{R}+1} \frac{P_{n}^{\prime}}{\sum_{n=1}^{N} P_{n}^{\prime}}+\delta(n-1) P_{1, \mathrm{LOS}},
$$

where $\delta(\cdot)$ is the Dirac delta function, $K_{R}$ is the Ricean Kfactor converted to linear scale, and $P_{1, \mathrm{LOS}}=K_{R} /\left(K_{R}+1\right)$.

According to step 7 of Section 7.5 in [1], ZOA is

$$
\theta_{n, \mathrm{ZOA}}^{\prime}=-\frac{\mathrm{ZSA} \ln \left(P_{n} / \max \left(P_{n}\right)\right)}{C_{\theta}},
$$

where $C_{\theta}=C_{\theta}^{\mathrm{NLoS}}\left(1.308+0.0339 K-0.0077 K^{2}+0.0002 K^{3}\right)$ for $\operatorname{LoS}$ and $C_{\theta}^{\mathrm{NLoS}}$ is given in Section 7.5 of [1]. 


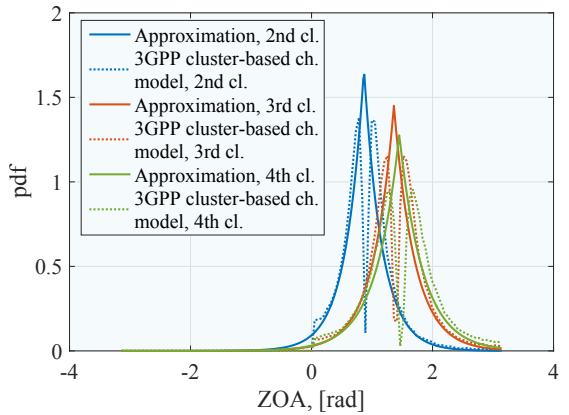

(a) ZOA marginal pdfs for clusters of 3GPP model

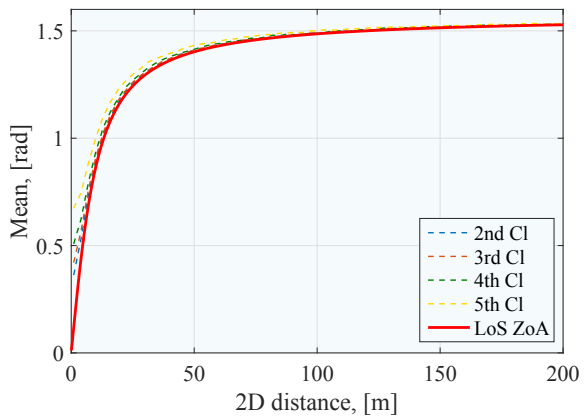

(b) Mean of ZOA

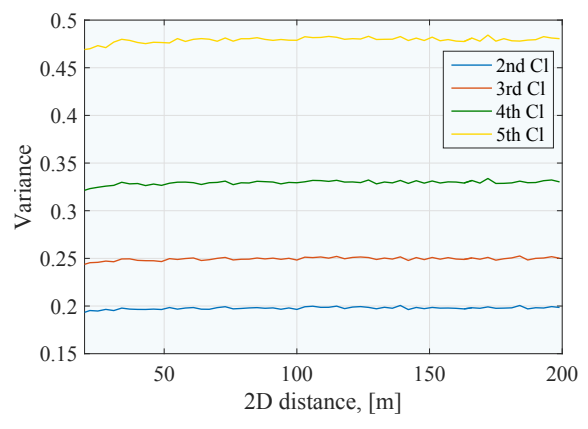

(c) Variance of ZOA

Figure 1: 3GPP ZOA characteristics and proposed approximations for $N=5$ clusters.

Finally, the ZOA in LoS conditions is

$$
\theta_{n, \mathrm{ZOA}}=\left[X_{n} \theta_{n, \mathrm{ZOA}}^{\prime}+Y_{n}\right]-\left[X_{1} \theta_{1, \mathrm{ZOA}}^{\prime}+Y_{1}-\theta_{\mathrm{LoS}, \mathrm{ZOA}}\right] \text {, }
$$

where $X_{n}$ is a discrete random variable (RV) that is uniformly distributed over $\{-1,1\}, Y_{n}$ follows a normal distribution with the zero mean and variance $(\mathrm{ZSA} / 7)^{2}$, and $\theta_{\mathrm{LoS}, \mathrm{ZOA}}$ is the $\mathrm{ZOA}$ of the LoS path calculated from the scenario geometry.

The generation procedure for the remaining parameters, such as ZOD and AOA/AOD, follows a similar approach and is presented in step 7 of Section 7.5 in [1].

\section{OUR PROPOSED APPROXIMATION}

In this section, we outline the proposed approximation for the important output parameters of the 3GPP 3D clusterbased model in case of LoS. For illustrative purposes, we concentrate on two parameters required in the performance evaluation of $5 \mathrm{G}$ mmWave cellular, accounting for random signal blockage: 1) ZOA and 2) received power share. We note that assuming the positions of blockers according to a Poisson point process (PPP), the AOA becomes irrelevant as the field of blockers is stochastically similar in any direction. The approach may also be used to approximate other parameters of the 3GPP cluster-based model. The settings used for the numerical examples are summarized in Table 1.

\subsection{GPP ZOA approximation}

We begin with ZOA, whose characteristics are presented in Fig. 1(a) for the heights of the mmWave access point (AP) and the UE equal to $h_{A}=10 \mathrm{~m}$ and $h_{U}=1.5 \mathrm{~m}$, respectively; we also focus on 5 strongest clusters for simplicity. The 2D distance, $x$, between the AP and the UE is set to $10 \mathrm{~m}, 40 \mathrm{~m}$, and $70 \mathrm{~m}$ for the illustration of 2-nd cluster, 3-rd cluster, and 4-th cluster, respectively. One may observe that ZOA can be well approximated by a Laplace distribution with the marginal probability density function (pdf) given by

$$
f_{\theta_{n}}(y)=\frac{1}{2 b_{n}} \exp \left(-\frac{\left|y-a_{n}\right|}{b_{n}}\right), n=2,3, \ldots,
$$

where $a_{n}$ and $b_{n}, n=2,3, \ldots, N$, are the distribution parameters, while $N$ is the number of clusters. While Fig. 1(a) shows some mismatch between the statistical pdf and the proposed approximation, the presented solution can be applied for the first-order analysis as further illustrated in Section 4.

We now proceed with calculating the parameters of the approximating Laplace distribution, since its mean and variance may depend on the scenario geometry (e.g., the distance between the nodes). To this end, Fig. 1(b) shows that the mean value for all the clusters, $a_{n}$, coincides with the $3 \mathrm{GPP} \operatorname{LoS}$ ZOA. Hence, the value of $a_{n}$ can be estimated as

$$
a_{n}=\frac{\pi}{2}-\arctan \left(\frac{h_{A}-h_{U}}{x}\right), n=2,3, \ldots, N .
$$

The second key parameter of the Laplace distribution is $b_{n}$, which is related to the distribution variance as $b_{n}=\sqrt{\sigma_{\theta_{n}}^{2} / 2}$.

Fig. 1(c) reports on the empirical variance of ZOA as a function of the $2 \mathrm{D}$ distance between the nodes. As can be observed, the variance for each of the clusters can be approximated by a constant value, from which we find $b_{n}$ as

$$
b_{1}=0, b_{2}=0.3146, b_{3}=0.3529, b_{4}=0.4056, b_{5}=0.4897 \text {. }
$$

Since both the approximating distribution and its key parameters have been established, we conclude that the approximation of the ZOA parameter of the 3GPP cluster-based model is constructed by combining (7), (8), and (9).

\subsection{GPP power share approximation}

The knowledge of ZOA allows to carefully model the process of clusters blockage by humans or other obstacles and thus estimate the probability for each of the clusters to be blocked. To evaluate the received power for certain clusters, we need to identify the share of power that they contribute at the $\mathrm{Rx}$. This will further allow for estimating the key metrics of interest, such as outage probability and link capacity.

Recall that according to 3GPP channel model outlined in Section 2, the received power from each cluster is calculated as a product of the received power determined by the averaged path loss and a random power share for each of the clusters normalized over the range $(0,1)$. Fig. 2(a) presents the pdfs of the power share values for first 5 clusters $\left(h_{A}=10 \mathrm{~m}\right.$ 


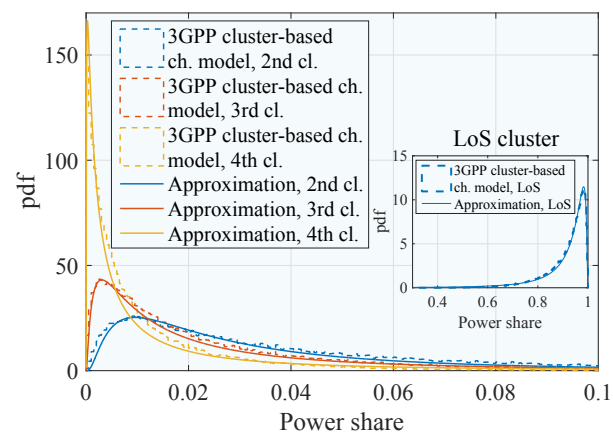

(a) Power share marginal pdfs for clusters

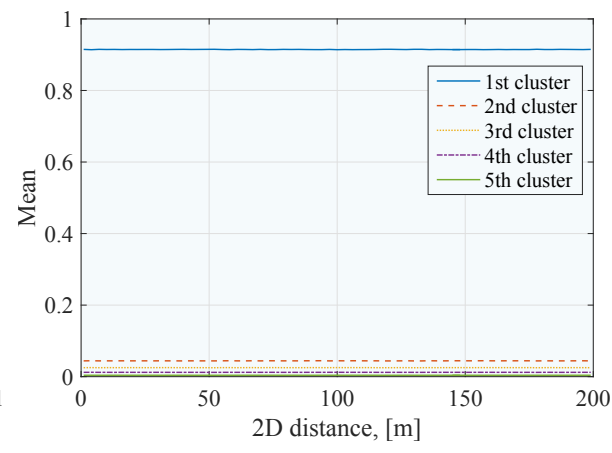

(b) Mean of power share

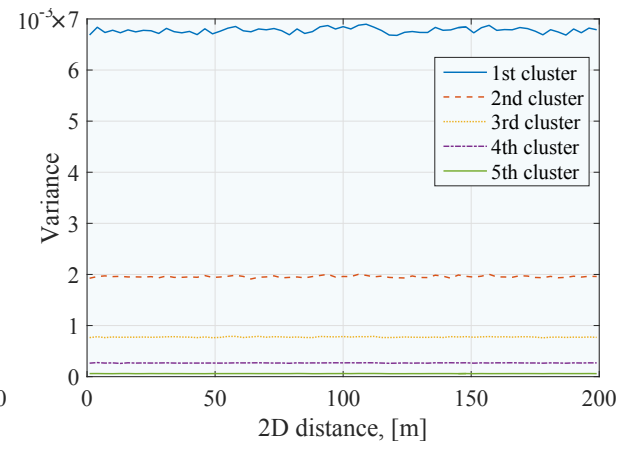

(c) Variance of power share

Figure 2: 3GPP power share characteristics and proposed approximations for $N=5$ clusters.

and $h_{U}=1.5 \mathrm{~m}$ ) as well as illustrates the fitting of empirical pdfs by a log-normal distribution for the 2-nd, 3-rd, 4-th, and 5 -th clusters (the 1-st cluster is a special case discussed below), in the following form

$f_{P_{s, n}}(z)=\frac{1}{z d_{n} \sqrt{2 \pi}} \exp \left(-\frac{\left(\ln z-c_{n}\right)^{2}}{2 d_{n}^{2}}\right), n=2,3, \ldots, N$,

where $c_{n}$ and $d_{n}$ are the parameters of the distribution.

The fraction of LoS power shown in Fig. 2(a) (1-st cluster) is always the cluster characterized by the strongest power. This value can be well approximated by a log-normal distribution with the argument $(1-x)$ and the parameters $\left(c_{1}, d_{1}\right)$,

$$
f_{P_{s, 1}}(z)=\frac{1}{(1-z) d_{1} \sqrt{2 \pi}} \exp \left(-\frac{\left(\ln (1-z)-c_{1}\right)^{2}}{2 d_{1}^{2}}\right) .
$$

The discretized version of the $\chi^{2}$ goodness-of-fit test has been applied to the distributions in Fig. 2(a) with the level of significance set to 0.95 , which shows that the hypothesis about the log-normal distribution does hold for the considered set of parameters. A similar conclusion applies to other system parameters as well. Therefore, the power shares can be approximated by the log-normal distribution and we continue by deriving its parameters.

We first notice that there is a direct relationship between the distribution parameters $\left(c_{n}\right.$ and $\left.d_{n}\right)$ and the estimated mean and variance $\left(\mu_{n}\right.$ and $\left.\sigma_{n}^{2}\right)$. Following the approach, we estimate the parameters of the power share distributions as

$$
\begin{aligned}
& c_{1}=-2.88, c_{2}=-3.55, c_{3}=-4.1, c_{4}=-4.98, c_{5}=-6.2, \\
& d_{1}=1.2, d_{2}=1.1, d_{3}=1.3, d_{4}=1.8, d_{5}=2.51
\end{aligned}
$$

In summary, the power share values can be well approximated by a combination of (10), (11), and (12). Hence, two key parameters of the 3GPP 3D cluster-based model to account for random blockage in 5G NR systems have been approximated by tractable analytical functions. The outlined approximations as well as the derived parameters $a_{n}, b_{n}, c_{n}$, and $d_{n}$ also hold for other environmental conditions $\left(h_{A}, h_{U}\right.$, etc.) as long as $N=5$.

\section{APPLICATION OF OUR MODEL}

In this section, we illustrate the applicability of the developed approximation to the analysis of $5 \mathrm{G}$ mmWave cellular systems. We particularly calculate the UE outage probability. We assume that the power share and ZOA do not depend on each other; we demonstrate with simulations that this assumption does not affect the outage probability. We then proceed by comparing the obtained results with those based on the algorithmic implementation of the 3GPP cluster-based channel model and UMi-street canyon channel model.

\subsection{Outage probability}

We consider the UE located at a fixed 2D distance of $x$ from its serving mmWave AP. The heights of AP and UE are assumed to be constant and set to $h_{A}$ and $h_{U}$. Blockers are represented by cylinders with the base radius of $r_{B}$ and the constant height of $h_{B}$. The blockers form a PPP with the density of $\lambda_{B}$. Owing to the random propagation environment, the ZOA, $\theta_{n}(x)$, and the received power share from each cluster, $P_{s, n}(x)$, are all RVs, where index 1 corresponds to the LoS path, $n=2,3, \ldots, N$ are clusters sorted in the increasing order according to their delays. The UE is always associated with the link having the strongest power. The UE is in outage when all the clusters are blocked or when the received power of the "best" cluster is below the target level.

Let $p_{n}$ be the probability that the $n$-th cluster of the link between the AP and the UE is blocked and first consider the blockage of the LoS path, $p_{1}$. Employing the results of [5], the LoS blockage probability is given by

$$
p_{1}(x)=1-e^{2 r_{B}\left(x \frac{h_{B}-h_{U}}{h_{A}-h_{U}}+r_{B}\right)} .
$$

In the 3GPP 3D cluster-based channel modeling algorithm, the exact location of the reflection point is not specified. However, by using ZOA, the blockage probability for the $n$-th cluster, $p_{n}(x)$, could be calculated as

$$
p_{n}(x)=\int_{-\pi}^{\pi} f_{\theta_{n}}(y ; x) q_{n}(y) d y,
$$


where $f_{\theta_{n}}(y ; x)$ is the distribution of the ZOA at the distance of $x$ approximated by the Laplace distribution with the parameters $a_{n}(x), b_{n}(x), n=2,3, \ldots, N$, all estimated from statistical data, $q_{n}(y)$ is the probability of blockage as a function of ZOA, which is given as

$$
q_{n}(y)=1-e^{-2 \lambda_{B} r_{B}\left(\tan y\left(h_{B}-h_{U}\right)+r_{B}\right)} .
$$

Substituting (15) and Laplace pdf (7) into (14), we obtain

$$
p_{n}(x)=\int_{-\pi}^{\pi} \frac{1-e^{-2 \lambda_{B} r_{B}\left(\tan y\left(h_{B}-h_{U}\right)+r_{B}\right)}}{2 b_{n} e^{\frac{\left|y-a_{n}(x)\right|}{b_{n}}}} d y,
$$

which can be evaluated numerically.

The received power of every cluster can be written as

$$
P_{n}=P_{s, n} 10^{\left(P_{T}-30-L\right) / 10}, n=1,2, \ldots, N,
$$

where $L$ is the path loss and $P_{T}$ is the transmit power. Using the 3GPP UMi LoS path loss model in (17), we arrive at

$$
P_{n}=P_{s, n} 10^{\left(P_{T}-30-32.4-20 \log _{10} f_{c}-21 \log _{10}\left(D_{3}\right)\right) / 10},
$$

where $D_{3}$ is the 3D distance between the AP and the UE.

Note that $P_{n}$ is a function of a RV. Therefore, by utilizing the RV transformation technique, the pdf of $P_{n}$ is given as

$$
\begin{gathered}
f_{P_{n}}(z ; x)=\frac{1}{10^{\left(P_{T}-30-32.4-20 \log _{10} f_{c}-21 \log _{10}\left(D_{3}\right)\right) / 10}} \\
f_{P_{s, n}}\left(\frac{P_{n}(x)}{10^{\left(P_{T}-30-32.4-20 \log _{10} f_{c}-21 \log _{10}\left(D_{3}\right)\right) / 10}}\right) .
\end{gathered}
$$

To evaluate the received power of the strongest cluster, we employ a weighted sum of the received power pdfs

$$
f_{P}(z ; x)=\sum_{n=1}^{N}\left[\left(1-p_{n}(x)\right) \prod_{j=1}^{n-1} p_{j}(x)\right] f_{P_{n}}(z ; x),
$$

where the weights are the probabilities of choosing cluster $n$.

We now proceed by obtaining the outage probability. Observe that outage occurs when the following mutually exclusive events happen: (i) all clusters are blocked or (ii) at least one cluster is non-blocked but the received signal falls below a certain threshold, $S_{T}$. The former probability is given as

$$
p_{O, 1}(x)=\prod_{n=1}^{N} p_{n}(x) .
$$

The probability that the received signal falls below $S_{T}$ is

$$
p_{O, 2}(x)=\int_{0}^{S_{T}} f_{P}(z ; x) d z,
$$

which leads to the outage probability in the following form

$$
p_{O}(x)=\prod_{n=1}^{N} p_{n}(x)+\int_{0}^{S_{T}} f_{P}(z ; x) d z .
$$

Table 1: Baseline system parameters

\begin{tabular}{ll}
\hline Parameter & Value \\
\hline Height of UE, $h_{U}$ and AP, $h_{A}$ & $1.5 \mathrm{~m}$ and $10 \mathrm{~m}$ \\
Height and radius of blocker, $h_{B}$ and $r_{B}$ & $1.7 \mathrm{~m}$ and $0.25 \mathrm{~m}$ \\
Number of clusters, $N$ & 5 \\
Transmit power, $P_{T}$ & $35 \mathrm{dBm}$ \\
SNR threshold & $3 \mathrm{~dB}$ \\
Carrier frequency, $f_{c}$ & $28 \mathrm{GHz}$ \\
Bandwidth & $1 \mathrm{GHz}$ \\
\hline
\end{tabular}

\subsection{Numerical results}

We continue with a numerical illustration of the outage probability under LoS conditions and human blockage environment as well as compare the results based on the proposed approximations against: (1) those given by an algorithmic implementation of the 3GPP cluster-based channel model [1] and (2) analytical model based on the 3GPP UMistreet canyon channel model [5]. In the second case, only the LoS path loss is considered for the analysis. In case of the LoS blockage, the received power is reduced by the human body loss $(20 \mathrm{~dB})$. The scenario parameters are given in Table 1.

Fig. 3 demonstrates the outage probability as a function of $2 \mathrm{D}$ distance between the AP and the UE and $\lambda_{B}=0.1$ for three approaches. We may observe four major intervals in this figure. For our set of parameters these are: (i) up to $28 \mathrm{~m}$; (ii) from 28 to $44 \mathrm{~m}$; (iii) from $44 \mathrm{~m}$ to $170 \mathrm{~m}$; (iv) over $170 \mathrm{~m}$.

The first interval shows outage probability close to 0 for all three models due to the short distance between AP and UE leading to a small LoS blockage probability and sufficiently high received power at the UE side even in case of blockage. In the second interval, both the simulation and approximation curves start increasing, since the chances of having the paths blocked are not negligible anymore. Meanwhile, the 3GPP UMi model underestimates the outage probability, as the modeled human body loss is not high enough to result in SNR being lower than the threshold value.

The third interval shows a considerable gap between the 3GPP UMi model and the cluster-based model together with its approximation. This is explained by increased blockage probability and only a single cluster when considering the 3GPP UMi model. In case of blockage of a single LoS cluster, the UE is considered in outage, while in cluster-based channel model the UE may communicate on different reflected clusters if some of them are not blocked and the received power is higher than the target level. Finally, the fourth interval illustrates a close match between all three models as the received clusters in the cluster-based models are contributing low power, so UE mainly employs the LoS cluster.

Fig. 4 shows the outage probability as a function of the density of blockers for two AP-UE 2D distances of $70 \mathrm{~m}$ and $49 \mathrm{~m}$. The approximation gives a tight match with the cluster-based model for a wide range of densities of blockers. 


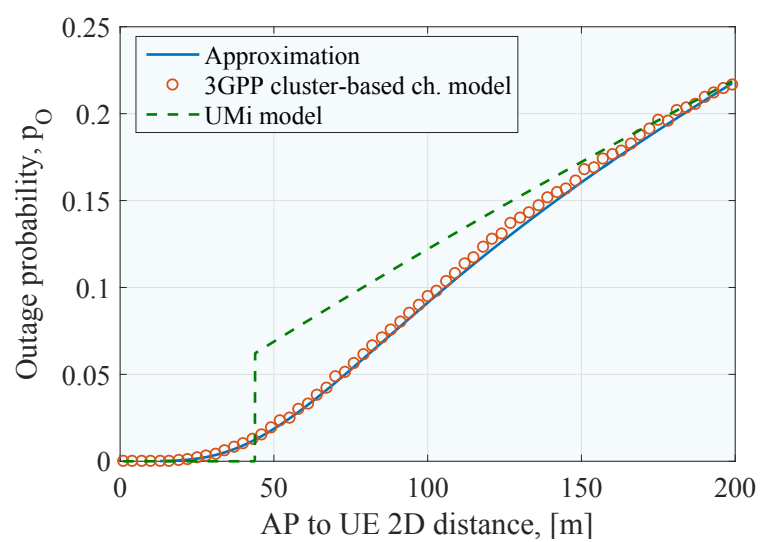

Figure 3: Outage probability $p_{O}$ vs. distance.

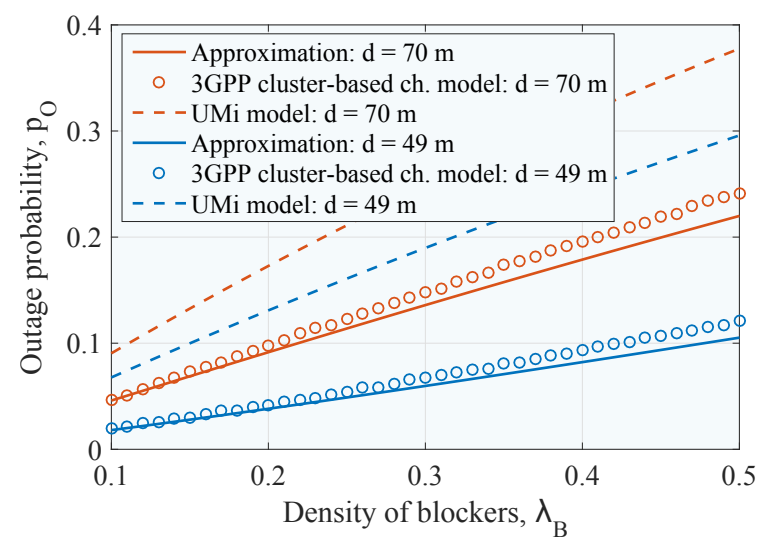

Figure 4: Outage probability $p_{O}$ as a function of $\lambda_{B}$.

Some deviation from the simulation plots at higher densities of blockers is due to imperfections of the approximated $\mathrm{ZoA}$ distribution. However, the 3GPP UMi model considerably overestimates the outage probability as compared to the cluster-based model, especially at higher distances. This is caused by increased blockage probability for higher densities of blockers. For the 3GPP UMi model, this growth leads to a significantly increased outage probability due to the absence of cluster diversity. Meanwhile, the outage probability for the cluster-based model increases much slower than that for the 3GPP UMi model due to the presence of alternative clusters that can be used in case of LoS blockage.

\section{CONCLUSIONS}

In this work, we developed analytically tractable approximations for the important parameters of the algorithmic 3D cluster-based channel model by 3GPP, including the zenith angle of arrival and the received power share of the clusters in LoS conditions. We demonstrated that these parameters can be well approximated by analytical distributions over a wide range of the environmental and system conditions. We also applied the proposed approximations to analytically characterize the outage probability in $5 \mathrm{G}$ mmWave cellular. We showed that the results obtained with our approximations closely match those directly produced by the algorithmic 3GPP 3D cluster-based model, in contrast to a simplistic model not capturing the multipath mmWave propagation.

The use of our proposed approximations improves the accuracy of known analytical approaches for the performance evaluation of NR systems. In addition, the outlined methodology can be further applied to construct more accurate approximations for both outlined and other important parameters of the 3GPP 3D cluster-based channel model, thus enabling more precise yet analytically tractable constructions for $5 \mathrm{G}$ and beyond mmWave networks.

\section{REFERENCES}

[1] 3GPP. 2018. Study on channel model for frequencies from 0.5 to $100 \mathrm{GHz}$ (Release 15). 3GPP TR 38.901 V15.0.0.

[2] M. R. Akdeniz, Y. Liu, M. K. Samimi, S. Sun, S. Rangan, T. S. Rappaport, and E. Erkip. 2014. Millimeter wave channel modeling and cellular capacity evaluation. IEEE Journal on Selected Areas in Communications 32, 6 (June 2014), 1164-1179.

[3] J. G. Andrews, T. Bai, M. N. Kulkarni, A. Alkhateeb, A. K. Gupta, and R. W. Heath. 2017. Modeling and Analyzing Millimeter Wave Cellular Systems. IEEE Transactions on Communications 65, 1 (January 2017), 403-430.

[4] M. Gapeyenko, A. Samuylov, M. Gerasimenko, D. Moltchanov, S. Singh, M. R. Akdeniz, E. Aryafar, N. Himayat, S. Andreev, and Y. Koucheryavy. 2017. On the Temporal Effects of Mobile Blockers in Urban MillimeterWave Cellular Scenarios. IEEE Transactions on Vehicular Technology 66, 11 (November 2017), 10124-10138.

[5] M. Gapeyenko, A. Samuylov, M. Gerasimenko, D. Moltchanov, S. Singh, E. Aryafar, S. Yeh, N. Himayat, S. Andreev, and Y. Koucheryavy. 2016. Analysis of Human Body Blockage in Urban Millimeter-Wave Wireless Communications Systems. In 2016 IEEE International Conference on Communications.

[6] IST-WINNER II. 2007. WINNER II Channel Models. Deliverable 1.1.2 v.1.2.

[7] C. De Looper. 2018. AT\&T names first three cities chosen to receive 5G networks by the end of 2018. https://www.digitaltrends.com/mobile/att5g-rollout/. Accessed on 14.08.18.

[8] G. R. MacCartney, T. S. Rappaport, and A. Ghosh. 2017. Base Station Diversity Propagation Measurements at $73 \mathrm{GHz}$ Millimeter-Wave for 5G Coordinated Multipoint (CoMP) Analysis. In IEEE Global Communications Workshops (GLOBECOM Wkshps). 1-7.

[9] F. Mademann. 2017. System architecture milestone of 5G Phase 1 is achieved. http://www.3gpp.org/NEWS-EVENTS/3GPP-NEWS/1930SYS_ARCHITECTURE. Accessed on 14.08.18.

[10] V. Petrov, M. A. Lema, M. Gapeyenko, K. Antonakoglou, D. Moltchanov, F. Sardis, A. Samuylov, S. Andreev, Y. Koucheryavy, and M. Dohler. 2018. Achieving End-to-End Reliability of Mission-Critical Traffic in Softwarized 5G Networks. IEEE fournal on Selected Areas in Communications 36, 3 (March 2018), 485-501.

[11] J. Smith. 2018. Verizon to use Samsung for first commercial $5 G$ network rollout. https:/www.zdnet.com/article/verizon-to-use-samsungrouters-for-initial-5g-rollout/. Accessed on 14.08.18.

[12] M. Zhang, M. Polese, M. Mezzavilla, S. Rangan, and M. Zorzi. 2017. Ns3 Implementation of the 3GPP MIMO Channel Model for Frequency Spectrum above $6 \mathrm{GHz}$. In Workshop on $n s-3$. 